\title{
AN IDENTITY FOR THE FIBONACCI AND LUCAS NUMBERS by DEREK JENNINGS
}

(Received 2 June, 1992)

In this paper we prove an identity between sums of reciprocals of Fibonacci and Lucas numbers. The Fibonacci numbers are defined for all $n \geq 0$ by the recurrence relation $F_{n+1}=F_{n}+F_{n-1}$ for $n \geq 1$, where $F_{0}=0$ and $F_{1}=1$. The Lucas numbers $L_{n}$ are defined for all $n \geq 0$ by the same recurrence relation, where $L_{0}=2$ and $L_{1}=1$. We prove the following identity.

THEOREM 1. For the Fibonacci and Lucas numbers we have

$$
\sum_{n=1}^{\infty} \frac{1}{\left(F_{2 n-1}\right)^{3}}=\sum_{n=1}^{\infty} \frac{1}{F_{2 n-1}}\left\{\sum_{n=1}^{\infty} \frac{1}{\left(F_{2 n-1}\right)^{2}}-5 \sum_{n=1}^{\infty} \frac{1}{\left(L_{2 n}\right)^{2}}\right\} \text {. }
$$

The above theorem is an immediate corollary of the following result.

TheOREM 2. For real $\alpha$ and $\beta$ such that $\alpha \beta=-1$ and $-1<\beta<0$ we have

$$
\sum_{n=1}^{\infty} \frac{1}{\left(\alpha^{2 n-1}-\beta^{2 n-1}\right)^{3}}=\sum_{n=1}^{\infty} \frac{1}{\left(\alpha^{2 n-1}-\beta^{2 n-1}\right)}\left\{\sum_{n=1}^{\infty} \frac{1}{\left(\alpha^{2 n-1}-\beta^{2 n-1}\right)^{2}}+\sum_{n=1}^{\infty} \frac{1}{\left(\alpha^{2 n}+\beta^{2 n}\right)^{2}}\right\} \text {. }
$$

Theorem 1 is proved by noting that $F_{n}=\left(\alpha^{n}-\beta^{n}\right) /(\alpha-\beta)$ and $L_{n}=\alpha^{n}+\beta^{n}$ where $\alpha=\frac{1}{2}(1+\sqrt{5})$ and $\beta=\frac{1}{2}(1-\sqrt{5})$, so that $\alpha$ and $\beta$ satisfy the conditions of Theorem 2 . Before we prove Theorem 2 we require two elementary lemmas.

Lemma 1. For $|q|<1$ we have

$$
\sum_{n=1}^{\infty} \frac{n q^{n}}{1+q^{n}}=\sum_{n=1}^{\infty} \frac{(2 n-1) q^{2 n-1}}{1-q^{2 n-1}}
$$

Proof. Logarithmically differentiate Euler's formula $\prod_{n=1}^{\infty}\left(1+q^{n}\right)\left(1-q^{2 n-1}\right)=1$.

Lemma 2. For $|q|<1$ we have

$$
\sum_{n=1}^{\infty} \frac{q^{n}}{\left(1+q^{n}\right)^{2}}=\sum_{n=1}^{\infty} \frac{n q^{n}}{1-q^{n}}-4 \sum_{n=1}^{\infty} \frac{n q^{2 n}}{1-q^{2 n}}
$$

Proof.

$$
\sum_{n=1}^{\infty} \frac{q^{n}}{\left(1+q^{n}\right)}=\sum_{n=1}^{\infty} \sum_{m=1}^{\infty}(-1)^{m+1} m q^{m n}=\sum_{n=1}^{\infty}\left\{\sigma^{o}(n)-\sigma^{\prime}(n)\right\} q^{n}
$$

where

$$
\sigma^{\prime}(n)=\sum_{d \mid n, d \text { odd }} \mathrm{d} \text { and } \sigma^{\prime \prime}(n)=\sum_{d \mid n, d \text { cren }} d .
$$
Now $\sigma^{\prime}(n)-\sigma^{\prime}(n)=\sigma(n)-4 \sigma(n / 2)$ where $\sigma(n)=\sum_{\left.d\right|_{n}} d$ and $\sigma(x)=0$ for non-integral $x$.
Therefore

$$
\sum_{n=1}^{\infty} \frac{q^{n}}{\left(1+q^{n}\right)^{2}}=\sum_{n=1}^{\infty}\left\{\sigma(n)-4 \sigma\left(\frac{n}{2}\right)\right\} q^{n}=\sum_{n=1}^{\infty} \frac{n q^{n}}{1-q^{n}}-4 \sum_{n=1}^{\infty} \frac{n q^{2 n}}{1-q^{2 n}} .
$$

Glasgow Math. J. 35 (1993) 381-384. 
To prove Theorem 2 we just note that it is an immediate corollary of the following result, with $q=\beta$, when $\alpha$ and $\beta$ satisfy the conditions of Theorem 2 .

THEOREM 3. For $|q|<1$ we have

$$
\sum_{n=1}^{\infty} \frac{q^{6 n-3}}{\left(1+q^{4 n-2}\right)^{3}}=\sum_{n=1}^{\infty} \frac{q^{2 n-1}}{1+q^{4 n-2}}\left\{\sum_{n=1}^{\infty} \frac{q^{4 n-2}}{\left(1+q^{4 n-2}\right)^{2}}-\sum_{n=1}^{\infty} \frac{q^{4 n}}{\left(1+q^{4 n}\right)^{2}}\right\} .
$$

Proof. We use Jacobi's triple product identity [1], which states that for complex $q$ and $z$ such that $|q|<1$ and $z \neq 0$ we have

$$
\prod_{n=1}^{\infty}\left(1-q^{2 n}\right)\left(1+z q^{2 n-1}\right)\left(1+z^{-1} q^{2 n-1}\right)=\sum_{n=-\infty}^{\infty} q^{n^{2}} z^{n}
$$

We now transform (1) by applying the following identities, which are effectively Chebyshev polynomials. For $n \geq 1$ and $z \neq 0$ we have

$$
z^{2 n}+\frac{1}{z^{2 n}}=\sum_{j=0}^{n}(-1)^{n+j} \frac{2 n}{n+j}\left(\begin{array}{c}
n+j \\
2 j
\end{array}\right)\left(z+z^{-1}\right)^{2 j}
$$

and for $n \geq 0, z \neq 0$ we have

$$
z^{2 n+1}+\frac{1}{z^{2 n+1}}=\sum_{j=0}^{n}(-1)^{n+j} \frac{2 n+1}{n+j+1}\left(\begin{array}{c}
n+j+1 \\
2 j+1
\end{array}\right)\left(z+z^{-1}\right)^{2 j+1} .
$$

From (1), with $x=z+z^{-1}$ and using the Chebshev polynomials to substitute for $z^{n}+z^{-n}$, then interchanging the order of summation we have

$$
\begin{aligned}
\prod_{n=1}^{\infty} & \left(1-q^{2 n}\right)\left(1+x q^{2 n-1}+q^{4 n-2}\right)=1+\sum_{n=1}^{\infty}\left\{z^{n}+\frac{1}{z^{n}}\right\} q^{n^{2}} \\
= & \sum_{j=0}^{\infty} \sum_{n=j}^{\infty}(-1)^{n+j} \frac{2 n}{n+j}\left(\begin{array}{c}
n+j \\
2 j
\end{array}\right) x^{2 j} q^{(2 n)^{2}}+\sum_{j=0}^{\infty} \sum_{n=j}^{\infty}(-1)^{n+j} \\
& \times \frac{2 n+1}{n+j+1}\left(\begin{array}{c}
n+j+1 \\
2 j+1
\end{array}\right) x^{2 j+1} q^{(2 n+1)^{2}}
\end{aligned}
$$

where $(2 n) /(n+j)$ is taken to be 1 when $n=j=0$. Equating the coefficients of $x^{3}$ in (2) gives

$$
\begin{aligned}
\prod_{n=1}^{\infty}\left(1-q^{2 n}\right) & \left(1+q^{4 n-2}\right)\left\{\left(\sum_{n=1}^{\infty} \frac{q^{2 n-1}}{1+q^{4 n-2}}\right)^{2}-3 \sum_{n=1}^{\infty} \frac{q^{2 n-1}}{1+q^{4 n-2}} \sum_{n=1}^{\infty}\right. \\
& \left.\times \frac{q^{4 n-2}}{\left(1+q^{4 n-2}\right)^{2}}+2 \sum_{n=1}^{\infty} \frac{q^{6 n-3}}{\left(1+q^{6 n-3}\right)^{3}}\right\}=\sum_{n=1}^{\infty}(-1)^{n+1} n(n+1)(2 n+1) q^{(2 n+1)^{2}}
\end{aligned}
$$

To evaluate the term on the right hand side of (3) we logarithmically differentiate the following famous theorem of Jacobi's [1]. For $|q|<1$ we have

$$
\prod_{n=1}^{\infty}\left(1-q^{n}\right)^{3}=\sum_{n=0}^{\infty}(-1)^{n}(2 n+1) q^{n(n+1) / 2}
$$

Then let $q:=q^{8}$ and multiply through by $q$ to give

$$
\sum_{n=1}^{\infty}(-1)^{n+1} n(n+1)(2 n+1) q^{(2 n+1)^{2}}=6 q \prod_{n=1}^{\infty}\left(1-q^{8 n}\right)^{3} \sum_{n=1}^{\infty} \frac{n q^{8 n}}{1-q^{8 n}} .
$$


We need two further results, (6) and (7), which are obtained by equating the coefficients of $x$ and $x^{2}$ respectively in (2). For the last equality in (6), we again use (4).

$$
\begin{aligned}
& \prod_{n=1}^{\infty}\left(1-q^{2 n}\right)\left(1+q^{4 n-2}\right) \sum_{n=1}^{\infty} \frac{q^{2 n-1}}{1+q^{4 n-2}}=\sum_{n=0}^{\infty}(-1)^{n}(2 n+1) q^{(2 n+1)^{2}}=q \prod_{n=1}^{\infty}\left(1-q^{8 n}\right)^{3} . \\
& \prod_{n=1}^{\infty}\left(1-q^{2 n}\right)\left(1+q^{4 n-2}\right) \frac{1}{2}\left\{\left(\sum_{n=1}^{\infty} \frac{q^{2 n-1}}{1+q^{4 n-2}}\right)^{2}-\sum_{n=1}^{\infty} \frac{q^{4 n-2}}{\left(1+q^{4 n-2}\right)^{2}}\right\}=\sum_{n=1}^{\infty}(-1)^{n+1} n^{2} q^{4 n^{2}} .
\end{aligned}
$$

Now let $z=-1$ in (1) and logarithmically differentiate. Then multiply through by $q$ to give

$$
\prod_{n=1}^{\infty}\left(1-q^{n}\right)\left(1-q^{2 n-1}\right)\left\{\sum_{n=1}^{\infty} \frac{n q^{n}}{1-q^{n}}+\sum_{n=1}^{\infty} \frac{(2 n-1) q^{2 n-1}}{1-q^{2 n-1}}\right\}=2 \sum_{n=1}^{\infty}(-1)^{n+1} n^{2} q^{n^{2}} .
$$

Then substitute for $\sum_{n=1}^{\infty} \frac{(2 n-1) q^{2 n-1}}{1-q^{2 n-1}}$ in (8), using Lemma 1, and let $q:=q^{4}$ to give

$$
\prod_{n=1}^{\infty}\left(1-q^{4 n}\right)\left(1-q^{8 n-4}\right) \sum_{n=1}^{\infty} \frac{n q^{4 n}}{1-q^{8 n}}=\sum_{n=1}^{\infty}(-1)^{n+1} n^{2} q^{4 n^{2}} .
$$

From Lemma 2 with $q:=q^{4}$ we have,

$$
\sum_{n=1}^{\infty} \frac{n q^{4 n}}{1-q^{8 n}}-3 \sum_{n=1}^{\infty} \frac{n q^{8 n}}{1-q^{8 n}}=\sum_{n=1}^{\infty} \frac{q^{4 n}}{\left(1+q^{4 n}\right)^{2}} .
$$

With the help of Euler's identity, $\prod_{n=1}^{\infty}\left(1+q^{n}\right)\left(1-q^{2 n-1}\right)=1$, we can combine (7), (9) and (10) to give,

$$
6 \sum_{n=1}^{\infty} \frac{n q^{8 n}}{1-q^{8 n}}=\left(\sum_{n=1}^{\infty} \frac{q^{2 n-1}}{1+q^{4 n-2}}\right)^{2}-\sum_{n=1}^{\infty} \frac{q^{4 n-2}}{\left(1+q^{4 n-2}\right)^{2}}-2 \sum_{n=1}^{\infty} \frac{q^{4 n}}{\left(1+q^{4 n}\right)^{2}} .
$$

Multiply (3) by $\sum_{n=1}^{\infty} \frac{q^{2 n-1}}{1+q^{4 n-2}}$. Substitute for $\sum_{n=1}^{\infty}(-1)^{n+1} n(n+1)(2 n+1) q^{(2 n+1)^{2}}$ from (5) and $\prod_{n=1}^{\infty}\left(1-q^{2 n}\right)\left(1+q^{4 n-2}\right) \sum_{n=1}^{\infty} \frac{q^{2 n-1}}{1+q^{4 n-2}}$ from (6). The term $q \prod_{n=1}^{\infty}\left(1-q^{8 n}\right)^{3}$ cancels. We then use (11) to substitute for $6 \sum_{n=1}^{\infty} \frac{n q^{8 n}}{1-q^{8 n}}$ in our new expression, and after the term in $\left(\sum_{n=1}^{\infty} \frac{q^{2 n-1}}{1+q^{4 n-2}}\right)^{3}$ conveniently cancels out we are left with Theorem 3 . So this completes the proof of Theorem 3 and hence that of Theorems 1 and 2 .

Conclusion. It was first shown by Landau [2] that

$$
\sum_{n=1}^{\infty} \frac{1}{F_{2 n-1}}=\frac{\sqrt{5}}{4} \theta_{2}^{2}\left(\frac{3-\sqrt{5}}{2}\right),
$$


where for $|q|<1$ we have,

Also it is known that

$$
\theta_{2}(q)=\sum_{n=-\infty}^{\infty} q^{(n+1 / 2)^{2}}
$$

$$
\sum_{n=1}^{\infty} \frac{1}{F_{2 n-1}^{3}}=\frac{5 \sqrt{5}}{32} \theta_{2}^{2}\left(\frac{3-\sqrt{5}}{2}\right)\left\{1-\theta_{4}^{4}\left(\frac{3-\sqrt{5}}{2}\right)\right\},
$$

where for $|q|<1$ we have,

$$
\theta_{4}(q)=\sum_{n=-\infty}^{\infty}(-1)^{n} q^{n^{2}}
$$

There are many other expressions for sums of reciprocals of Fibonacci and Lucas numbers in terms of the theta functions. See [3] for more examples. Also there are other known polynomial identities between these sums. For example

and

$$
\sum_{n=1}^{\infty} \frac{1}{L_{n}^{2}}=2\left(\sum_{n=1}^{\infty} \frac{1}{L_{2 n}}\right)^{2}+\sum_{n=1}^{\infty} \frac{1}{L_{2 n}},
$$

$$
4\left(\sum_{n=1}^{\infty} \frac{1}{F_{2 n-1}}\right)^{2}=5 \sum_{n=1}^{\infty} \frac{1}{L_{n}^{2}}+3 \sum_{n=1}^{\infty} \frac{1}{F_{n}^{2}} .
$$

Of course we could prove Theorem 1 by showing that,

$$
\sum_{n=1}^{\infty} \frac{1}{F_{2 n-1}^{2}}-5 \sum_{n=1}^{\infty} \frac{1}{L_{2 n}^{2}}=\frac{5}{8}\left\{1-\theta_{4}^{4}\left(\frac{3-\sqrt{5}}{2}\right)\right\},
$$

and then use results (12) and (13). However, such a proof would be less direct than the proof given.

\section{REFERENCES}

1. G. H. Hardy and E. M. Wright, An introduction to the theory of numbers (Clarendon Press, Oxford, 1938).

2. E. Landau, Sur la Serie des Invers de Nombres de Fibonacci. Bull. Soc. Math. France, 27 (1899), 298-300.

3. J. M. Borwein and P. B. Borwein, Pi and the AGM (Wiley 1987).

\section{Barford Close}

North Millers Dale

Chandlers Ford

HAMPSHIRE

$5051 \mathrm{TH}$

ENGLAND 